\title{
ASYMPTOTIC BEHAVIOR OF OSCILLATING RADIAL SOLUTIONS TO CERTAIN NONLINEAR EQUATIONS, PART II*
}

\author{
CHANGFENG GUI ${ }^{\dagger}$, XUE LUO ${ }^{\ddagger}$, AND FENG ZHOU $§$
}

Key words. Asymptotic behavior, Allen-Cahn equations and thin film problems, oscillation.

AMS subject classifications. 35B45, 35J60, 35B05

1. Introduction. In this note, we consider the following nonlinear problem

$$
\left\{\begin{array}{l}
u^{\prime \prime}+\frac{n-1}{r} u^{\prime}+\beta^{2} u+f(u)=0, \quad r>0 \\
u(r) \rightarrow 0, \quad \text { as } r \rightarrow \infty,
\end{array}\right.
$$

where $n \geq 2$,

$$
f \in C^{1, \sigma}\left(-\delta_{0}, \delta_{0}\right), \text { for some } \delta_{0}>0, \sigma>0 \text { and } f(0)=f^{\prime}(0)=0 .
$$

The main goal of this note is to show the asymptotic behavior of solutions of (1) and improve the results in [6]. In [6], one of the following conditions

$$
\left\{\begin{array}{c}
a): f(u) \in C^{1, \sigma}\left(-\delta_{0}, \delta_{0}\right), f(0)=f^{\prime}(0)=0, \\
\sigma>\frac{2}{n-1} \text { if } n>3, \text { or } \\
b): f(u) \in C^{2, \sigma}\left(-\delta_{0}, \delta_{0}\right), f(0)=f^{\prime}(0)=f^{\prime \prime}(0)=0, \\
\sigma>0 \text { if } n=3, \text { or } \\
c): f(u) \in C^{3, \sigma}\left(-\delta_{0}, \delta_{0}\right), f(0)=f^{\prime}(0)=f^{\prime \prime}(0)=f^{(3)}(0)=0, \\
\sigma>0 \text { if } n=2,
\end{array}\right.
$$

is assumed, and the existence and asymptotic behavior of oscillatory radial solutions are proven. We replace the conditions by a more general condition (2), therefore equation (1) can be applied to Allen-Cahn equation

$$
\triangle u+u-u^{3}=0, \quad x \in \mathbb{R}^{n},
$$

for all $n \geq 2$, and thin film problems

$$
u^{\prime \prime}+\frac{n-1}{r} u^{\prime}=f(u) \quad \text { in } \mathbb{R}_{+}, \quad u(0)=\alpha>0, u^{\prime}(0)=0,
$$

where $f \in C^{1}(0, \infty)$ satisfies the following general conditions:

(i) $f$ has a single zero $t_{0}$ in $(0, \infty)$ satisfying $f^{\prime}\left(t_{0}\right)<0$;

\footnotetext{
${ }^{*}$ Received September 8, 2009; accepted for publication January 5, 2010. This work is partially supported by NSF, NSFC and CSC.

${ }^{\dagger}$ Department of Mathematics, U-9, University of Connecticut, Storrs, CT 06269, USA (gui@math. uconn.edu).

${ }^{\ddagger}$ Department of Mathematics, East China Normal University, Shanghai, 200241 China (luoxue 0327@163.com).

$\S$ Department of Mathematics, East China Normal University, Shanghai, 200241 China (fzhou @math.ecnu.edu.cn).
} 
(ii) $f$ is nonincreasing near 0 and $\lim _{t \rightarrow 0^{+}} f(t)=\infty$.

These two equations appear in several applications in mechanics and physics. Interested readers can refer [1], [2]-[4], [10], [13], [14], etc. for more detailed physics background. Some recent mathematical analysis can be found in $[5,6,7,8,9,11,12]$ and the references therein.

REMARK 1. We note that oscillating solutions to thin film problems may not always exist when $n=2$. It is shown in [9] that the unique solution either oscillates or increases to infinity as $r$ goes to infinity. The existence of non-blowup solution may depend on the initial value and the nonlinear term $f$.

In [9], a recent paper concerning thin film problems (see also [12]), it was proven that in dimension $N \geq 3$, for each $\alpha \in\left(0, t_{0}\right)$, (4) has a unique positive solution $u_{\alpha}$. Moreover, $u_{\alpha}$ oscillates around the constant $t_{0}$. It is also shown that there exists a singular (or so-called rupture) radial solution $u_{0}(r)$ to (4) such that $u_{0} \in C\left(\mathbb{R}^{N}\right)$, $u_{0}(0)=0, u_{0}(r)>0$ for $r \in(0, \infty)$ and $f\left(u_{0}\right) \in L_{l o c}^{1}\left(\mathbb{R}^{N}\right)$. Moreover, any singular radial solution to (4) is oscillatory around $t_{0}$ and converges to $t_{0}$ as $r \rightarrow \infty$.

It is natural to ask whether we can obtain more accurate asymptotic behaviors of the radial solutions. The similar question also arises in the study of Allen-Cahn equation (3).

The main result in this note is stated below:

TheOrem 1. Assume $f$ satisfies (2) and equation (1) has a solution $u(r)$, then $u(r)$ is oscillating and $|u(r)| \leq C r \frac{1-n}{2}$. Furthermore,

$$
u(r)=r^{\frac{1-n}{2}}(A \sin (\beta r)+B \cos (\beta r)+o(1)),
$$

as $r \rightarrow \infty$, for some constants $A, B$.

This note is organized as follows: In Section 2, the proof of the main result will be given; Section 3 will be devoted to applying the main result to Allen-Cahn equations and thin film equations to get a more accurate asymptotic behaviors.

2. Proof of Theorem 1. First, we claim that the solution, if it converges to 0 as $r \rightarrow+\infty$, must be oscillatory.

Lemma 1. Assume that $f$ satisfies condition (2). Then the solution to equation (1) is oscillatory around 0.

Proof. Suppose that the solution is not oscillatory. Without loss of generality, we may assume $u(r)>0, r>\tilde{r}$, for some $\tilde{r}>0$. By (2), we may choose $\tilde{r}$ so large that $\frac{f(u(r))}{u(r)}>-\frac{\beta^{2}}{2}$, for $r>\tilde{r}$. Then $u(r)$ is decreasing for $r>\tilde{r}$, by the maximum principle. Then we define $\omega\left(r, r_{0}\right):=\frac{u\left(r+r_{0}\right)}{u\left(r_{0}\right)}$, for $r \geq 0$ and any fixed $r_{0}>\tilde{r}$. Then $\omega\left(r, r_{0}\right)$ satisfies

$$
\omega_{r r}+\frac{n-1}{r+r_{0}} \omega_{r}+\beta^{2} \omega+\frac{f\left(u\left(r+r_{0}\right)\right)}{u\left(r_{0}\right)}=0,
$$

for $r \geq 0$. Letting $r_{0} \rightarrow+\infty$, we know that $\omega\left(r, r_{0}\right) \rightarrow \omega(r, \infty)$ in $C^{2}[0, \infty)$ and

$$
\left\{\begin{array}{l}
\omega_{r r}(r, \infty)+\beta^{2} \omega(r, \infty)=0 \\
\omega(0, \infty)=1,0 \leq \omega(r, \infty) \leq 1
\end{array}\right.
$$


for all $r>\tilde{r}$. This contradicts the Sturm-Liouville Theorem. Similarly we can exclude the case $u(r)<0$ for $r$ sufficiently large.

Without loss of generality, we may assume that $|u(r)| \leq \delta_{0}, \forall r>0$. Let $s_{k}$ be the $k^{\text {th }}$ zero of $u(r)$ and $r_{k}$ is the maximum point of $|u|$ in $\left(s_{k}, s_{k+1}\right)$. Set $m_{k}:=\left|u\left(r_{k}\right)\right|$. We claim that $m_{k}$ is decreasing in $k$, when $k>\tilde{k}$, for some $\tilde{k}>0$, and tends to 0 as $k$ goes to infinity. In fact, on the one hand, we multiply $u^{\prime}$ on both sides of the first equation of $(1)$, then

$$
\left\{\frac{1}{2} u^{\prime 2}+\frac{1}{2} \beta^{2} u^{2}+\int_{0}^{u} f(s) d s\right\}^{\prime}=-\frac{n-1}{r} u^{\prime 2} \leq 0,
$$

which implies that $\frac{1}{2} u^{\prime 2}+\frac{1}{2} \beta^{2} u^{2}+\int_{0}^{u} f(s) d s$ is decreasing in $r>0$. Take $r=$ $r_{k}$ and $r_{k+1}$, we get

$$
\begin{aligned}
\frac{1}{2} \beta^{2} m_{k+1}^{2}+ & \int_{0}^{m_{k+1}} f(s) d s \\
\int_{m_{k}}^{m_{k+1}} f(s) d s & \leq \frac{1}{2} \beta^{2} m_{k}^{2}+\int_{0}^{m_{k}} f\left(m_{k}^{2}-m_{k+1}^{2}\right) .
\end{aligned}
$$

On the other hand, we have $f \in C^{1, \sigma}\left(-\delta_{0}, \delta_{0}\right), \sigma>0$, then

$$
\left|\int_{m_{k}}^{m_{k+1}} f(s) d s\right| \leq \int_{m_{k}}^{m_{k+1}}|f(s)| d s \leq C \int_{m_{k}}^{m_{k+1}}|s|^{1+\sigma} d s \leq C\left(m_{k+1}^{\sigma+2}-m_{k}^{\sigma+2}\right)
$$

if $m_{k+1}>m_{k}$. Suppose that no matter how large $k$ is, there always exist some $k$ such that $m_{k+1}>m_{k}$, then

$$
C\left(m_{k}^{2+\sigma}-m_{k+1}^{2+\sigma}\right) \leq-\left|\int_{m_{k}}^{m_{k+1}} f(s) d s\right|<\int_{m_{k}}^{m_{k+1}} f(s) d s \leq \frac{1}{2} \beta^{2}\left(m_{k}^{2}-m_{k+1}^{2}\right) .
$$

Hence

$$
m_{k+1}^{2}-m_{k}^{2} \leq C\left(m_{k+1}^{2+\sigma}-m_{k}^{2+\sigma}\right)
$$

where $C$ is independent of $k$. This contradicts with the fact that $m_{k} \rightarrow 0$, as $k \rightarrow+\infty$. Therefore, $m_{k+1} \leq m_{k}$, for $k>\tilde{k}$.

Next, we state a simple fact as a lemma, which will be used later.

LEMMA 2. The problem,

$$
\phi^{\prime \prime}(s)+p(s) \phi(s)=0
$$

where $0<a^{2}<p(s)<b^{2}<\infty, a, b$ are some constants, for $s \in I$, where $I$ is a finite or infinite interval, have solutions with

$$
\frac{\pi}{b} \leq s_{k+1}-s_{k} \leq \frac{\pi}{a} \quad \text { and } \quad \frac{\pi}{b} \leq t_{k+1}-t_{k} \leq \frac{\pi}{a}
$$

for $s_{k}, t_{k} \in I$, where $s_{k}$ is the $k^{\text {th }}$ zero of $\phi$ in $I, t_{k}$ is the maximum point of $|\phi|$ in the interval $\left(s_{k}, s_{k+1}\right)$.

Proof. The lemma basically follows from the Sturm Comparison Theorem. For the convenience, we present a direct proof here. Without loss of generality, we may 
assume $\phi\left(t_{k}\right)>0$. We claim $s_{k+1}-t_{k} \leq \frac{\pi}{2 a}$. Choose a solution $v(r)=\cos \left(a\left(r-t_{k}\right)\right)$ of $v^{\prime \prime}+a^{2} v=0$. If $s_{k+1}>t_{k}+\frac{\pi}{2 a}$, then

$$
\int_{t_{k}}^{\frac{\pi}{2 a}+t_{k}}\left(v \phi^{\prime}-\phi v^{\prime}\right)^{\prime} d r=a \phi\left(t_{k}+\frac{\pi}{2 a}\right)>0
$$

On the other hand, by equations we obtain

$$
\int_{t_{k}}^{\frac{\pi}{2 a}+t_{k}}\left(v \phi^{\prime}-\phi v^{\prime}\right)^{\prime} d r=\int_{t_{k}}^{\frac{\pi}{2 a}+t_{k}}\left(a^{2}-p(r)\right) \phi v d r<0
$$

This is a contradiction and proves the claim. Similarly, we can show $t_{k}-s_{k} \leq \frac{\pi}{2 a}$. Then $s_{k+1}-s_{k} \leq \frac{\pi}{a}$ and $t_{k+1}-t_{k} \leq \frac{\pi}{a}$. Similar arguments also show $s_{k+1}-s_{k} \geq \frac{\pi}{b}$ and $t_{k+1}-t_{k} \geq \frac{\pi}{b}$. $\square$

With the simple observation, we see that making the following transformation

$$
u(r)=r^{\frac{1-n}{2}} \phi(r),
$$

equation (1) can be rewritten as the equation of $\phi$ in the form of (7), where $p(s)=$ $\beta^{2}-\frac{(n-1)(n-3)}{4} s^{-2}+\frac{f(u)}{u}=\beta^{2}+O\left(s^{\alpha}\right)$, for some $\alpha<0$, when $s$ sufficiently large, since $f \in C^{1, \sigma}\left(-\delta_{0}, \delta_{0}\right)$, for some $\delta_{0}>0$ and $|u(r)|<C r^{\frac{1-n}{2}+\epsilon}$ in [6]. It is easy to see that the $\mathrm{k}^{\text {th }}$ zero of $u$ is also that of $\phi$, denoted as $s_{k}$, but the maximum point $r_{k}$ of $|u|$ in $\left(s_{k}, s_{k+1}\right)$ are different from that $t_{k}$ of $|\phi|$.

Next lemma is devoted to estimate $\phi(s)$ and $\phi^{\prime}(s)$ in the interval $\left(t_{k}, t_{k+1}\right)$.

Lemma 3. Assume $p(s)=\beta^{2}+O\left(s^{\alpha}\right)$ for some $\alpha<0$ and $s_{k}, t_{k}$ are defined as before. Then for $k$ large enough, there holds

$$
t_{k+1}-t_{k}=\frac{\pi}{\beta}+O\left(k^{\alpha}\right), \quad t_{k}-s_{k}=\frac{\pi}{2 \beta}+O\left(k^{\alpha}\right),
$$

and

$$
\begin{aligned}
& \frac{\phi(s)}{\phi\left(t_{k}\right)}=\cos \left(\beta\left(s-t_{k}\right)\right)+O\left(k^{\alpha}\right), \quad t_{k}<s<t_{k+1} \\
& \frac{\phi^{\prime}(s)}{\phi\left(t_{k}\right)}=-\beta \sin \left(\beta\left(s-t_{k}\right)\right)+O\left(k^{\alpha}\right), \quad t_{k}<s<t_{k+1} .
\end{aligned}
$$

Proof. When $k$ is large enough, we choose $C$ so that $a^{2}=\beta^{2}-C k^{\alpha}>0$, $b^{2}=\beta^{2}+C k^{\alpha}>0$ and $a^{2}<p(s)<b^{2}, t_{k}<s<t_{k+1}$. By the Sturm Comparison Theorem or similar proof of Lemma 2, we have

$$
\frac{\phi(s)}{\phi\left(t_{k}\right)} \leq \cos \left(\sqrt{\beta^{2}-C k^{\alpha}}\left(s-t_{k}\right)\right), \quad t_{k}<s<s_{k+1}
$$

and

$$
\frac{\phi(s)}{\phi\left(t_{k}\right)} \geq \cos \left(\sqrt{\beta^{2}+C k^{\alpha}}\left(s-t_{k}\right)\right), \quad t_{k}<s<t_{k}+\frac{\pi}{2 \sqrt{\beta^{2}+C k^{\alpha}}}
$$


In particular, $\frac{\pi}{2 \sqrt{\beta^{2}+C k^{\alpha}}} \leq s_{k+1}-t_{k} \leq \frac{\pi}{2 \sqrt{\beta^{2}-C k^{\alpha}}}$. Hence, $s_{k+1}-t_{k}=\frac{\pi}{2 \beta}+O\left(k^{\alpha}\right)$. Similarly,

$$
\begin{array}{ll}
\frac{\phi(s)}{\phi\left(t_{k}\right)} \leq \cos \left(\sqrt{\beta^{2}-C k^{\alpha}}\left(t_{k}-s\right)\right), \quad s_{k}<s<t_{k}, \\
\frac{\phi(s)}{\phi\left(t_{k}\right)} \geq \cos \left(\sqrt{\beta^{2}+C k^{\alpha}}\left(t_{k}-s\right)\right), \quad t_{k}-\frac{\pi}{2 \sqrt{\beta^{2}+C k^{\alpha}}}<s<t_{k},
\end{array}
$$

and $t_{k}-s_{k}=\frac{\pi}{2 \beta}+O\left(k^{\alpha}\right)$. Hence, $t_{k+1}-t_{k}=\frac{\pi}{\beta}+O\left(k^{\alpha}\right)$.

On the other hand, multiply $\phi^{\prime}(s)$ on both sides of (7) and integrate from $t_{k}$ to $t_{k+1}$, we get

$$
\begin{gathered}
\int_{t_{k}}^{t_{k+1}} p(s) \phi(s) \phi^{\prime}(s) d s=0 \\
\Rightarrow \quad \phi^{2}\left(t_{k+1}\right)=\phi^{2}\left(t_{k}\right)\left(1+O\left(k^{\alpha}\right)\right),
\end{gathered}
$$

since $p(s)=\beta^{2}+O\left(k^{\alpha}\right)>0$, for $k$ sufficiently large. Combining (11)-(15) for the interval $\left(t_{k}, t_{k+1}\right)$, we conclude $(9)$.

To conclude (10), we multiply the equation (7) by $\phi^{\prime}(s)$ and integrate from $t_{k}$ to $s$ to get

$$
\frac{1}{2}\left(\phi^{\prime}(s)\right)^{2}+\int_{t_{k}}^{s} p(s) \phi^{\prime}(s) \phi(s) d r=0, \quad t_{k}<s<s_{k+1} .
$$

Without loss of generality, suppose $\phi(s)>0, s \in\left(t_{k}, s_{k+1}\right)$. By (16), we have $\phi^{\prime}(s)<$ 0 , for $s \in\left(t_{k}, s_{k+1}\right)$, then

$$
\begin{aligned}
\frac{1}{2}\left(\phi^{\prime}(s)\right)^{2} & \leq\left(\beta^{2}+C k^{\alpha}\right) \int_{t_{k}}^{s}\left(-\phi^{\prime}(s)\right) \phi(s) d s \\
& =\left(\beta^{2}+C k^{\alpha}\right) \frac{1}{2}\left(\phi^{2}\left(t_{k}\right)-\phi^{2}(s)\right), \quad t_{k}<s<s_{k+1} .
\end{aligned}
$$

Then $\frac{1}{2}\left(\phi^{\prime}(s)\right)^{2}=\frac{1}{2} \beta^{2} \phi^{2}\left(t_{k}\right) \sin ^{2}\left(\beta\left(s-t_{k}\right)\right)+O\left(k^{\alpha}\right), t_{k}<s<s_{k+1}$, by using the lower bound of $p(s)$ together. Combining the equality and its counterpart for $\left(s_{k+1}, t_{k+1}\right)$, we get $(10)$.

Following is a refined version of Lemma 4.3 in [6], which is key to our proof of the main result.

LEMMA 4. Let $\left\{a_{k}\right\}$ be a sequence of nonincreasing positive numbers satisfing

$$
a_{k} \geq p \sum_{i=k}^{\infty} a_{i} h(i)
$$

for some positive $p$, where $h(i)=i^{-1}\left(1+O\left(i^{\gamma}\right)\right), \gamma<0$. Then, for some positive constant $C$, there holds

$$
a_{k} \leq C k^{-p},
$$

for $k$ sufficiently large. 
Proof. Define $A_{k}:=\sum_{i=k}^{\infty} a_{i} h(i)$, then

$$
\begin{array}{r}
A_{k}-A_{k+1}=a_{k} h(k) \geq p h(k) A_{k} \\
\Rightarrow \quad \frac{A_{k}}{A_{k+1}} \geq \frac{1}{1-p h(k)} \geq 1+p h(k),
\end{array}
$$

for $k$ large enough. Let $N_{0}$ large fixed. For any integer $N>N_{0}$, we have

$$
\begin{aligned}
& \frac{A_{N_{0}}}{A_{N}} \geq \prod_{k=N_{0}}^{N-1}(1+p h(k)) \\
\Rightarrow & \ln \left(A_{N_{0}}\right)-\ln \left(A_{N}\right) \geq \sum_{k=N_{0}}^{N-1}\left(p h(k)+O\left(h^{2}(k)\right)\right) \geq p \ln N+O(1),
\end{aligned}
$$

since $h(i)=i^{-1}\left(1+O\left(i^{\gamma}\right)\right), \gamma<0$. Therefore, $A_{N}<C N^{-p}$. With this fact that $\left\{a_{k}\right\}$ is a nonincreasing positive sequence, we obtain

$$
A_{k} \geq A_{k}-A_{2 k+1}=p \sum_{i=k}^{2 k} a_{i} h(i) \geq p a_{2 k} \sum_{i=k}^{2 k} h(i) \geq \frac{p \ln 2}{2} a_{2 k},
$$

since $\sum_{i=k}^{2 k} h(i) \rightarrow \ln 2$, as $k \rightarrow \infty$ and $h(i)>0$, for $i$ large enough. Therefore, $a_{2 k} \leq$ $C A_{k}<C k^{-p}$, for $k$ sufficiently large, where $C>0$ is just dependent of $p$. $\square$

Now it's time to refine the decay rate of $u(r)$ as $O\left(r^{\frac{1-n}{2}}\right)$ with Lemma 4 and complete the proof of Theorem 1.

Proof of Theorem 1. Let $\tilde{m}_{k}:=u\left(t_{k}\right)$, while $m_{k}:=u\left(r_{k}\right)$, where $t_{k}, r_{k}$ are the maximum points of $|\phi|,|u|$ in $\left(s_{k}, s_{k+1}\right)$, respectively. First, with Lemma 4 , we could get a sharper estimate of $\tilde{m}_{k}$.

Denote $F(u)=-\frac{1}{2} \beta^{2} u^{2}-\int_{0}^{u} f(s) d s$, then

$$
\begin{aligned}
F(0)-F\left(\tilde{m_{k}}\right) & =\frac{1}{2} \beta^{2} \tilde{m}_{k}^{2}+\int_{0}^{\tilde{m_{k}}} f(s) d s \leq \frac{1}{2} \beta^{2} \tilde{m}_{k}^{2}+C \tilde{m}_{k}^{2+\sigma} \\
& \leq \frac{1}{2} \beta^{2} \tilde{m}_{k}^{2}+C k^{\tilde{\delta}},
\end{aligned}
$$

where $\tilde{\delta}=(2+\sigma)\left(\frac{1-n}{2}+\epsilon\right)<0$, for $k$ large enough. In fact, the inequality above follows by $f \in C^{1, \sigma}\left(-\delta_{0}, \delta_{0}\right)$ and the fact shown in [6] that $|u(r)|<C r^{\frac{1-n}{2}+\epsilon}$, for any $\epsilon>0$. Furthermore,

$$
\begin{aligned}
F(0)-F\left(\tilde{m_{k}}\right) & =\lim _{N \rightarrow \infty}\left[F\left(\tilde{m_{N}}\right)-F\left(\tilde{m_{k}}\right)\right] \\
& =\lim _{N \rightarrow \infty}\left[-\frac{1}{2}\left(u^{\prime 2}\left(t_{N}\right)-u^{\prime 2}\left(t_{k}\right)\right)+\int_{t_{k}}^{t_{N}} \frac{n-1}{r}\left|u^{\prime}(r)\right|^{2} d r\right], \text { by }(6) \\
& =\frac{1}{2} u^{\prime 2}\left(t_{k}\right)+\lim _{N \rightarrow \infty} \int_{t_{k}}^{t_{N}} \frac{n-1}{r}\left|u^{\prime}(r)\right|^{2} d r \\
& \geq \lim _{N \rightarrow \infty} \int_{t_{k}}^{t_{N}} \frac{n-1}{r}\left|u^{\prime}(r)\right|^{2} d r .
\end{aligned}
$$


With the fact that

$$
u^{\prime}(r)=\frac{1-n}{2} r^{-\frac{1+n}{2}} \phi(r)+r^{\frac{1-n}{2}} \phi^{\prime}(r),
$$

we have

$$
\begin{aligned}
& \int_{t_{k}}^{t_{k+1}} \frac{n-1}{r}\left|u^{\prime}(r)\right|^{2} d r \\
= & \int_{0}^{\frac{\pi}{\beta}+O\left(k^{\alpha}\right)} \frac{n-1}{\frac{k \pi}{\beta}+O\left(k^{\alpha}\right)} \\
& \cdot\left(\frac{1-n}{2}\left(t_{k}+x\right)^{-\frac{1+n}{2}} \phi\left(t_{k}+x\right)+\left(t_{k}+x\right)^{\frac{1-n}{2}} \phi^{\prime}\left(t_{k}+x\right)\right)^{2} d x \\
= & \int_{0}^{\frac{\pi}{\beta}+O\left(k^{\alpha}\right)} \frac{n-1}{\frac{k \pi}{\beta}+O\left(k^{\alpha}\right)} \tilde{m}_{k}^{2} \beta^{2} \sin ^{2}(\beta x)\left(1+O\left(k^{\alpha}\right)\right) d x \\
= & (n-1) \tilde{m}_{k}^{2} \beta^{2} \frac{1}{2 k}\left(1+O\left(k^{\alpha}\right)\right),
\end{aligned}
$$

by (9), (10) and (19). Here $\alpha<0$ changes from line to line. Combining (17), (18) and (20), we have

$$
(n-1) \sum_{i=k}^{\infty} \tilde{m}_{i}{ }^{2} h(i)-C k^{\tilde{\delta}} \leq \tilde{m}_{k}{ }^{2},
$$

where $h(i)=\frac{1}{i}\left(1+O\left(i^{\alpha}\right)\right)$.

Now, we apply Lemma 4 to (21) to get a more accurate decay rate of $\tilde{m}_{k}$. We claim that there exists $\tilde{C}>0$, such that

$$
a_{k}+b_{k} \geq(n-1) \sum_{i=k}^{\infty}\left(a_{i}+b_{i}\right) h(i),
$$

where $a_{k}=\tilde{m}_{k}^{2}, b_{k}=\tilde{C} k^{\tilde{\delta}}$. Indeed, from (21), we know that (22) is true, as long as there exists $\tilde{C}>0$ such that

$$
-C k^{\tilde{\delta}} \geq(n-1) \sum_{i=k}^{\infty} b_{i} h(i)-b_{k},
$$

where $b_{k}=\tilde{C} k^{\tilde{\delta}}$. In fact,

$$
\begin{aligned}
(n-1) \sum_{i=k}^{\infty} b_{i} h(i)-b_{k} & =\tilde{C}\left[(n-1) \sum_{i=k}^{\infty} i^{\tilde{\delta}} h(i)-k^{\tilde{\delta}}\right] \\
& =\tilde{C}\left[(n-1) \sum_{i=k}^{\infty} i^{-1+\tilde{\delta}}\left(1+O\left(i^{\alpha}\right)\right)-k^{\tilde{\delta}}\right] \\
& =\tilde{C}\left(\frac{1-n}{\tilde{\delta}}-1+o(1)\right) k^{\tilde{\delta}} \\
& \leq-C k^{\tilde{\delta}}
\end{aligned}
$$


provided $\frac{1-n}{\tilde{\delta}}-1<0$, i.e. $\tilde{\delta}:=(2+\sigma)\left(\frac{1-n}{2}+\epsilon\right)<1-n$. Since $\epsilon>0$ is arbitrary, then we can choose $\epsilon$ small enough, such that $\tilde{\delta}<1-n$. Now, we let $\left\{\tilde{a_{k}}\right\}$, where $\tilde{a_{k}}=a_{k}+b_{k}$, be the nonincreasing sequence in Lemma 4. By Lemma 4, we get

$$
\begin{aligned}
\tilde{a_{k}} & \leq C k^{1-n} \\
\Rightarrow \quad a_{k} & \leq C k^{1-n}-\tilde{C} k^{\tilde{\delta}} \leq C k^{1-n},
\end{aligned}
$$

since $\tilde{\delta}<1-n$. Therefore,

$$
\tilde{m}_{k} \leq C k^{\frac{1-n}{2}} .
$$

Since $m_{k}=O\left(\tilde{m}_{k}\right)$, we obtain $m_{k}<C k^{\frac{1-n}{2}}$, moreover, $|u(r)|<C r^{\frac{1-n}{2}}$. Then (5) follows immediately. $\mathrm{Q}$

3. Applications to Allen-Cahn equations and thin film equations. Finally, we apply the main theorem to two typical problems, namely Allen-Cahn equation and thin film problems, which have been investigated in [9], [6], [12], etc.

We first consider the radial solution to Allen-Cahn equation

$$
\left\{\begin{array}{l}
u^{\prime \prime}+\frac{n-1}{r} u^{\prime}-F^{\prime}(u)=0, \quad r=|x|, x \in \mathbb{R}^{n}, \\
u(0)=u_{0},\left|u_{0}\right|<1,
\end{array}\right.
$$

where $n \geq 2, F(u) \in C^{2, \sigma}\left(-\delta_{0}, \delta_{0}\right)$, for some $\delta_{0}>0, \sigma>0$, and satisfies

$$
\left\{\begin{array}{l}
F^{\prime}(1)=F^{\prime}(-1)=0, F(1)=F(-1)=0 \\
F(u)>0 \quad \text { if }|u|<1 \\
F^{\prime}(0)=0, F^{\prime \prime}(0)<0 \\
F^{\prime}(u)<0 \quad \text { if } 0<u<1, F^{\prime}(u)>0 \quad \text { if }-1<u<0 .
\end{array}\right.
$$

The existence of the oscillatory radial solution to Allen-Cahn Equation with initial value $\left|u_{0}\right|<1$ has been shown in Prop 3.1 and Prop 3.2, [5]. We can obtain the asymptotic behavior of the solution as follows.

Theorem 2. Assume $f(u)=F^{\prime \prime}(0) u-F^{\prime}(u)$ satisfies condition (2). Then when $\left|u_{0}\right|<1$, the solution $u(r)$ satisfies $|u(r)| \leq C r^{\frac{1-n}{2}}$. Furthermore,

$$
u(r)=r^{\frac{1-n}{2}}\left(A \sin \left(\sqrt{-F^{\prime \prime}(0)} r\right)+B \cos \left(\sqrt{-F^{\prime \prime}(0)} r\right)+o(1)\right),
$$

as $r \rightarrow \infty$, for some constants $A, B$.

In particular, for the typical Allen-Cahn equation $u^{\prime \prime}+\frac{1-n}{r} u^{\prime}+u-u^{3}=0$ in $\mathbb{R}^{2}$, we have $|u(r)| \leq C r^{-\frac{1}{2}}$ and $s_{k+1}-s_{k}=\pi+O\left(k^{\alpha}\right)$, for some $\alpha<0$, where $s_{k}$ is the $k^{\text {th }}$ zero of the solution.

Now, we consider the thin film problem

$$
\left\{\begin{array}{l}
u^{\prime \prime}+\frac{n-1}{r} u^{\prime}=g(u) \quad r>0 \\
u(0)=u_{0}>0, u^{\prime}(0)=0
\end{array}\right.
$$

where the nonlinear term $g(u)$ satisfies

$$
\left\{\begin{array}{l}
g^{\prime}(1)<0, g(1)=0 \\
g(u)>0 \text { for } 0<u<1, g(u)<0 \text { for } u>1 .
\end{array}\right.
$$


Let $v(r)=u(r)-1$, then it satisfies

$$
v^{\prime \prime}+\frac{n-1}{r} v^{\prime}-g^{\prime}(1) v+f(v)=0, r>0,
$$

where $f(v)=-g(1+v)+g^{\prime}(1) v$.

From [6], we know that when $n \geq 3$, there always exists a radial solution, for $u_{0} \in(0,1)$, which oscillates around 1 . However, when $n=2$, we have the solution either asymptotic to 1 or blow up to $+\infty$ as $r$ tends to $\infty$.

THEOREM 3. Assume $g(u)$ satisfies condition (26) and $f(v)=-g(1+v)+g^{\prime}(1) v$ satisfies condition (2). For $n \geq 3$, when $u_{0} \in(0,1)$, the solution $u(r)$ to (25) satisfies $|u(r)| \leq C r^{\frac{1-n}{2}}$. Furthermore,

$$
u(r)=r^{\frac{1-n}{2}}\left(A \sin \left(\sqrt{-g^{\prime}(1)} r\right)+B \cos \left(\sqrt{-g^{\prime}(1)} r\right)+o(1)\right),
$$

as $r \rightarrow \infty$, for some constants $A, B$.

When it comes to $n=2$, we have to pose an extra condition on $g$ to guarantee the existence of oscillatory solutions. Let $G(u)=\int_{1}^{u} g(s) d s$, then $G(u)$ is nonincreasing for $u>1$ and nondecreasing for $u<1$.

THEOREM 4. When $n=2, u_{0} \in(0,1)$, assume $g$ satisfies condition (26) and $\lim _{u \rightarrow+\infty} G(u)=-\infty$, additionally, $f(v)$ satisfies condition (2). Then there exists an oscillatory radial solution $u(r)$ with $|u(r)| \leq C r^{-\frac{1}{2}}$. Furthermore,

$$
u(r)=r^{-\frac{1}{2}}\left(A \sin \left(\sqrt{-g^{\prime}(1)} r\right)+B \cos \left(\sqrt{-g^{\prime}(1)} r\right)+o(1)\right),
$$

as $r \rightarrow \infty$, for some constants $A, B$.

Acknowledgements. The research of Gui is partially supported by National Science Foundation Grant DMS 0500871 and an Oversea Cooperation Fund of National Science Foundation of China. Part of this work was completed during Luo's visit to the University of Connecticut with the financial support of CSC. She would like to thank the Department of Mathematics of the University of Connecticut for its warm hospitality. The research of Zhou is supported by NSFC (No.10971067). The authors would also like to thank the referee for many valuable comments and suggestions.

\section{REFERENCES}

[1] S. Allen and J. W. Cahn, A Microscopic Theory for Antiphase Boundary Motion and its Application to Antiphase Domain Coarsening, Acta. Metall., 27 (1979), pp. 1084-1095.

[2] A. L. Bertozzi and M. C. Pugh, Long-wave Instabilities and Saturation in Thin Film Equations, Comm. Pure Appl. Math., 51 (1998), pp. 625-661.

[3] A. L. Bertozzi and M. C. Pugh, Finite-time Blow-up of Solutions of Some Long-wave Unstable Thin Film Equations, Indiana Univ. Math. J., 49 (2000), pp. 1323-1366.

[4] J. P. Burelbach, S. G. Bankoff and S. H. Davis, Nonlinear Stability of Evaporating/Condensing Liquid Films, J. Fluid Mech., 195 (1988), pp. 463-494.

[5] C. F. Gui, Lecture Notes on Allen-Cahn Equation and Its Generation, in preparation.

[6] C. F. Gui And F. Zhou, Asymptotic Behavior of Oscillating Radial Solutions to Certain Nonlinear Equations, Methods and Applications of Analysis, 15:3 (2008), pp. 285-296.

[7] H. X. Guo, Z. M. Guo And K. Li, Positive solutions of a Semilinear Elliptic Equation with Singular Nonlinearity, J. Math. Anal. Appl., 323 (2006), pp. 344-359. 
[8] Z. M. Guo And J. C. WeI, Symmetry of Nonnegative Solutions of a Semilinear Elliptic Equation with Singular Nonlinearity, Proc. Royal. Soc. Edinburgh. Section A, 137 (2007), pp. 963-994.

[9] Z. M. Guo, D. Ye And F. Zhou, Existence of Singular Positive Solutions for Some Semilinear Elliptic Equations, Pacific J. Math., 236:1 (2008), pp. 57-51.

[10] C. C. Hwang, C. K. Lin And W. Y. Uen, A Nonlinear Three-dimensional Rupture Theory of Thin Liquid Films, J. Colloid Interf. Sci., 190 (1997), pp. 250-252.

[11] H. Q. JiAng AND F. H. Lin, Zero Set of Sobolev Functions with Negative Power of Integrability, Chinese Ann. Math. Ser. B, 25 (2004), pp. 65-72.

[12] H. Q. JIANG AND W. M. NI, On steady states of van der Waals force driven thin film equations, European J. Applied Math., 18 (2007), pp. 1-28.

[13] R. S. Laugesen And M. C. Pugh, Properties of Steady States for Thin Film Equations, Europ. J. Appl. Math., 11 (2000), pp. 293-351.

[14] R. S. Laugesen And M. C. Pugh, Linear Stability of Steady States for Thin Film and CahnHilliard Type Equations, Arch. Rat. Mech. Anal., 154 (2000), pp. 3-51. 